Article

\title{
Fully Symmetric Relativistic Quantum Mechanics and Its Physical Implications
}

\author{
Bao D. Tran and Zdzislaw E. Musielak*(D)
}

Departmemt of Physics, University of Texas at Arlington, Arlington, TX 76019, USA; bao.tran3@mavs.uta.edu

* Correspondence: zmusielak@uta.edu

\begin{abstract}
A new formulation of relativistic quantum mechanics is presented and applied to a free, massive, and spin-zero elementary particle in the Minkowski spacetime. The reformulation requires that time and space, as well as the timelike and spacelike intervals, are treated equally, which makes the new theory fully symmetric and consistent with the special theory of relativity. The theory correctly reproduces the classical action of a relativistic particle in the path integral formalism, and allows for the introduction of a new quantity called vector-mass, whose physical implications for nonlocality, the uncertainty principle, and quantum vacuum are described and discussed.
\end{abstract}

Keywords: relativistic quantum mechanics; generalized Klein-Gordon equation; path integral formulation; nonlocality; quantum measurement; uncertainty principle; quantum vacuum

Citation: Tranl, B.D.; Musielak, Z.E.

Fully Symmetric Relativistic

Quantum Mechanics and Its Physical Implications. Mathematics 2021, 9 , 1213. https://doi.org/10.3390/ math9111213

Academic Editor: Rami Ahmad El-Nabulsi

Received: 2 May 2021

Accepted: 25 May 2021

Published: 27 May 2021

Publisher's Note: MDPI stays neutral with regard to jurisdictional claims in published maps and institutional affiliations.

\section{Introduction}

Relativistic quantum mechanics (RQM) primarily concerns free relativistic fields [1,2] described by the Klein-Gordon [3,4], Dirac [5], Proca [6], and Rarita-Schwinger [7] wave equations, whereas the fundamental interactions and their unification are considered by the gauge invariant quantum field theory (QFT) [8,9]. The above equations of RQM are invariant with respect to all transformations that form the Poincaré group $\mathcal{P}=S O(3,1) \otimes_{s}$ $T(3+1)$, where $S O(3,1)$ is a non-invariant Lorentz group of rotations and boosts and $T(3+1)$ an invariant subgroup of spacetime translations, and this structure includes reversal of parity and time [10]. Bargmann and Wigner [11] used the representations of $\mathcal{P}$ [12] to obtain the Bargmann-Wigner equations, which reduce to the RQM equations under special conditions. Different methods of deriving the RQM equations were proposed by using either symmetry properties [13] or the irreducible representations (irreps) of $T(3+1)$ and the resulting eigenvalue equations [14,15].

There were several attempts to generalize the RQM equations, specifically, the KleinGordon [16-19], Dirac [20-25], or both Klein-Gordon and Dirac [26-28] and other [29] equations. Physical motivations for these generalizations were different and they range from including some supersymmetry effects to unification of leptons and quarks and accounting for different masses of the three generations of elementary particles. Recently, the Dirac equation was generalized to include chiral symmetry and it was shown that the correct identification of chiral bases may explain the small masses of neutrinos and be used to predict a new massive particle of dark matter [30].

As required by special theory of relativity (STR), time and space must be treated equally, which means that all basic equations of RQM are symmetric in their time and space derivatives. In STR, the timelike and spacelike intervals are also equally allowed, even so, RQM has been formulated using the timelike intervals only [1,2]; known exceptions are some tachyonic versions of RQM and QFT [31,32]. The main objective of this paper is to reformulate RQM by taking into account both timelike and spacelike intervals and explore the physical implications of the developed theory. The presented theory is developed for a free, massive, and spin-zero elementary particle in the Minkowski spacetime. We follow 
Wigner [12] and define an elementary (quantum) particle as an object whose wavefunction transforms as one of the irreps of $\mathcal{P}[10]$.

The presence of the spacelike intervals in our theory modifies its linear momentum that, in addition to its timelike eigenvalues, also has spacelike eigenvalues. As a result, the STR energy-momentum relationship where its scalar-mass is modified by an additional term with its vector-mass, which only affects the spatial distribution of particle's wavefunction. The physical consequences of the presence of the vector-mass in the energymomentum relationship are far reaching, starting with the modifications to the mass term in the Klein-Gordon equation, and then leading to nonlocality, some modifications of the uncertainty principle, and a novel picture of vacuum. Moreover, the developed RQM theory preserves causality, is self-consistent, and is formulated using the path integral formalism, which correctly reproduces the classical action of a relativistic particle; however, the theory does not allow for tachyons $[31,32]$. The theory is not a hidden-variable theory (e.g., [33]) and its physical interpretation is consistent with the Copenhagen interpretation of quantum mechanics (QM) [34,35].

The paper is structured as follows: the generalized eigenvalue equations are presented and discussed in Section 2; the basic postulates used to reformulate RQM are described in Section 3; the generalized Klein-Gordon equation and its solutions are given in Section 4; in Section 5, completeness of Hilbert space and causality of the theory are demonstrated; in Section 6, the theory is formulated by using the path integral approach; physical implications of the theory are given in Section 7; and conclusions are in Section 8.

\section{Generalized Eigenvalue Equations}

The basis of our reformulation of RQM is a set of eigenvalue equations for the fourmomentum operator $\mathcal{K}_{\mu}=i \partial_{\mu}$ acting on a relativistic quantum state $|\phi\rangle$ in the Minkowski spacetime, with the metric tensor $\eta_{\mu \nu}$ of positive time signature. The eigenvalue equations are given by

$$
\mathcal{K}_{\mu}|\phi\rangle=\left(P_{\mu}+V_{\mu}\right)|\phi\rangle,
$$

where $P_{\mu}$ and $V_{\mu}$ are timelike and spacelike eigenvalues of $\mathcal{K}_{\mu}$, respectively, and $P_{\mu} P^{\mu}=m^{2}$, $V_{\mu} V^{\mu}=-\tilde{m}^{2}$ and $P_{\mu} V^{\mu}=0$, with $m$ being a scalar mass and $\tilde{m}$ being a new quantity called here "vector-mass" (see Section 3 for discussion). The eigenfunction $\phi$ can be either scalar, or vector, or any higher rank tensor, or spinor wavefunction in order to be consistent with the irreps of $\mathcal{P}[10,12]$.

As shown previously [14,15], the eigenvalue equations for $\phi$ being a scalar wavefunction represent the necessary conditions that this function transforms as one of the irreps of $T(3+1) \in \mathcal{P}$; here Equation (1) is a generalization of the previous results, which are recovered when $V_{\mu}=0$. A new RQM is formulated by using the above eigenvalue equations, which means that time and space, as well as the timelike $\left(P_{\mu}\right)$ and spacelike $\left(V_{\mu}\right)$ intervals, are treated equally as required by STR.

In this paper, we consider only scalar wavefunctions and follow Wigner $[10,12]$ to identify an elementary particle with one of the irreps of $\mathcal{P}$. In other words, the scalar wavefunction $\phi$ represents an elementary particle if, and only if, the wavefunction satisfies the above generalized eigenvalue equations.

The eigenvalue equations given by Equation (1) are expressed in terms of a general coordinate basis that is not necessarily orthonormal. Therefore, we formulate our theory in the local orthonormal basis, called tetrads, which are shown to be of great advantage in the development of our new RQM. The tetrads are defined as local coordinate transformations from a global curvilinear coordinate system to a locally flat orthogonal coordinate system that is considered as the local inertial frame of an observer in the Minkowski spacetime.

We used Greek indices for global coordinate components and Latin indices to denote the components of local tetrad coordinates with $\eta_{a b}=g_{\mu \nu} U_{a}^{\mu} U_{b}^{v}$ and $g_{\mu \nu}$ being the metric tensor of the global coordinate system. The tetrads are defined as $U_{\mu}^{a}=\frac{\partial \xi^{a}}{\partial x^{\mu}}$, where $\xi^{a}$ is 
the local coordinate function. Then, the invariant spacetime element $d s^{2}$ of the Minkowski metric and the eignevalue equations in the new local tetrad basis can be written as

$$
\mathrm{d} s^{2}=g_{\mu \nu} \mathrm{d} x^{\mu} \mathrm{d} x^{\nu}=\eta_{a b} \mathrm{~d} \xi^{a} \mathrm{~d} \xi^{b},
$$

and

$$
\mathcal{K}_{a}|\phi\rangle=\left(P_{a}+V_{a}\right)|\phi\rangle=K_{a}|\phi\rangle .
$$

We express the contravariant components of the four-vectors $P$ and $V$ in the local basis as $P=\{E, \boldsymbol{P}\}$ and $V=\{U, V\}$ that locally satisfy the following conditions: $P_{a} P^{a}=$ $E^{2}-\boldsymbol{P} \cdot \boldsymbol{P}=m^{2}, V_{a} V^{a}=U^{2}-\boldsymbol{V} \cdot \boldsymbol{V}=-\tilde{m}^{2}$ and $P_{a} V^{a}=0$, where $m$ and $\tilde{m}$ are the so-called scalar and vector mass, respectively. It must be noted that both $m$ and $\tilde{m}$ are Lorentz-invariant constants, and that $U=\boldsymbol{P} \cdot \boldsymbol{V} / E$.

Let $\beta=P / E$ be the relativistic velocity of an elementary particle, which allows writing $P=\{E, \boldsymbol{\beta} E\}, V=\{\boldsymbol{\beta} \cdot \boldsymbol{V}, \boldsymbol{V}\}$, and $K=P+V=\{E+\boldsymbol{\beta} \cdot \boldsymbol{V}, \boldsymbol{V}+\boldsymbol{\beta} E\}$. We refer to the vector $K$ as the four-mass vector and use it to define the eigenstate wavefunction of a relativistic quantum particle. The four-vector $P$ provides the energy-momentum relationship of STR, which is used in standard RQM. However, the presence of the four-vector $V$ modifies this relationship to become

$$
\left(E^{2}-|\boldsymbol{P}|^{2}\right)+\left(U^{2}-|\boldsymbol{V}|^{2}\right)=m^{2}-\tilde{m}^{2}=M^{2},
$$

which is the energy-momentum relationship for a massive and spin-zero quantum particle used in the reformulated RQM. This energy-momentum relationship reduces to that of STR, which is the basis for standard RQM, when $V=0$.

It must be also noted that $M^{2}$ is not characterized as the mass of the particle, as in this reformulated RQM, a particle of definite mass and position in space must be in a superposition of different vector-mass $\tilde{m}$, and that the scalar-mass $m$ represents is responsible for the particle's world-line to be confined within the light cone. On the other hand, the vector-mass $k$ does not play any role in the motion of the particle, even if its squared magnitude exceeds that of the scalar-mass, because of its spacelike origin. Therefore, the theory developed in this paper only allows for particles with positive $m^{2}$ term, which rules out tachyons and tachyonic fields [31,32] as unphysical within the framework of this theory.

The origin of the four-vector $V$ is the generalized eigenvalue equations given by Equation (1), and the above results show that the vector-mass $\tilde{m}$ is associated with the presence of $V$. In our reformulated RQM, $m$ is responsible for the time evolution of the wavefunction $\phi$, as in standard RQM, while $\tilde{m}$ affects only the spatial distribution of the wavefunction $\phi$, which makes our reformulated RQM a fully symmetric theory that accounts for both the timelike and spacelike intervals. Let us now describe the basic postulates of our new theory.

\section{Basic Postulates of New Theory}

Our reformulation of RQM is based on the following postulates:

1. The canonical commutation relation of the conjugate operators $\left(\xi^{a}, \mathcal{K}_{b}\right)$ is given as

$$
\left[\xi^{a}, \mathcal{K}_{b}\right]=-i \delta_{b}^{a} .
$$

2. The particle's relativistic three-velocity, $\beta=P / E$, is the boost parameter of a proper Lorentz transformation

$$
\Lambda_{\beta}=\left(\begin{array}{cc}
\gamma & \gamma \boldsymbol{\beta}^{\top} \\
\gamma \boldsymbol{\beta} & \gamma \mathrm{I}+\frac{\gamma^{2}}{1+\gamma} \mathrm{B}^{2}
\end{array}\right),
$$


where $\boldsymbol{\beta}^{\top}$ is a row vector, the symbol $\top$ denotes matrix transposition, the $3 \times 3$ identity matrix is represented by $\mathrm{I}, \gamma=\left(1-|\boldsymbol{\beta}|^{2}\right)^{-1 / 2}$ is the Lorentz factor with $|\boldsymbol{\beta}|<1$, and

$$
\mathrm{B}^{2}=\beta \beta^{\top}-|\beta|^{2} \mathrm{I}
$$

with

$$
\mathrm{B}=\left(\begin{array}{ccc}
0 & \beta_{z} & -\beta_{y} \\
-\beta_{z} & 0 & \beta_{x} \\
\beta_{y} & -\beta_{x} & 0
\end{array}\right)
$$

From this, we have $E=\gamma m$ as the usual definition for the energy of a particle of scalar-mass $m$, and the new energy contribution related to vector-mass $k$ is defined as $\boldsymbol{V}=\mathrm{L} \boldsymbol{k}$, where $\mathrm{L}$ is given by

$$
\mathrm{L}=\gamma \mathrm{I}+\frac{\gamma^{2}}{1+\gamma} \mathrm{B}^{2}=\mathrm{I}+\frac{\gamma^{2}}{1+\gamma} \boldsymbol{\beta} \boldsymbol{\beta}^{\top} .
$$

Thus, the four-mass vector in the rest frame, i.e., $\boldsymbol{\beta}=\mathbf{0}$, is simply $K=k=\{m, \boldsymbol{k}\}$.

3. Let $\mathcal{U}_{\beta}$ be an operator identified as a unitary representation of the Lorentz boost that acts on the state vector $|k\rangle=|m, k\rangle$ in a Hilbert space

$$
|m, \boldsymbol{k} ; \boldsymbol{\beta}\rangle \equiv \mathcal{U}_{\boldsymbol{\beta}}|m, \boldsymbol{k}\rangle \text {. }
$$

Here, the states $|m, k\rangle$ are the so-called inertial states describing a particle at rest, with $m$ and $\boldsymbol{k}$ forming the four-mass vector $K$, and the velocity $\boldsymbol{\beta}=\mathbf{0}$. A particle can be boosted to a moving frame with $\beta \neq 0$, so the state of a moving particle is labeled as $|m, k ; \boldsymbol{\beta}\rangle$. The dual states of the inertial states are called the spacetime states and they are represented by the vector $|\xi\rangle$, or $|\tau, \xi\rangle$, in a rest-frame of an observer.

The states of all $m$ and $k$ form a complete basis of the relativistic quantum Hilbert space of a particle at rest, and the vector $|\xi\rangle$, or $|\tau, \xi\rangle$, also form a complete basis for the spacetime location of the particle (see Section 5). The fact that neither $m$ nor $k$ are quantities with set up values (see Section 6) makes the presented RQM a significantly different theory than standard RQM, and its physical implications are far reaching (see Section 7).

4. The inertial state $|m, k\rangle$ is an eigenvector of the operators $\mathcal{K}_{a}$ such that

$$
\mathcal{K}_{a}|m, \boldsymbol{k}\rangle=i \partial_{a}|m, \boldsymbol{k}\rangle=k_{a}|m, \boldsymbol{k}\rangle \text {, }
$$

with the eigenvalues $k=\{m, \boldsymbol{k}\}$. It follows that $k_{a} k^{a}=m^{2}-|\boldsymbol{k}|^{2}=m^{2}-\tilde{m}^{2}=M^{2}$. It must be also noted that the vector-mass is completely independent from the scalar-mass.

5. The wavefunction of a quantum particle at rest, with the scalar-mass $m$ and vectormass $k$, expressed in the spacetime basis is given by

$$
\langle\tau, \boldsymbol{\xi} \mid m, \boldsymbol{k}\rangle=\frac{e^{-i(m \tau-\boldsymbol{k} \cdot \boldsymbol{\xi})}}{(2 \pi)^{2}}
$$

where $\tau=\xi^{0}$, and the Lorentz-transformed wavefunction of a particle moving with a well-defined velocity $\beta$ is given by

$$
\langle\tau, \boldsymbol{\xi} \mid m, \boldsymbol{k} ; \boldsymbol{\beta}\rangle=\frac{e^{-i \tau(E+\boldsymbol{\beta} \cdot \boldsymbol{V})} e^{i \boldsymbol{\xi} \cdot(\boldsymbol{V}+\boldsymbol{\beta} E)}}{(2 \pi)^{2}} .
$$

The state in Equation (12) describes a particle at rest, nevertheless, the wavefunction seems to be propagating in the direction of $k$. However, a more careful look shows that it is not propagating, but instead it is the winding of the complex wavefunction. Thus, there is nothing actually moving though space and the only way to assign 
motion to the particle is to change the motion of the inertial frame of the observer relative to the frame of the particle through a Lorentz boost, as given in Equation (13).

6. The timelike differential operator $Q_{t}=t^{a} \mathcal{K}_{a}$, where $t^{a}$ is a timelike vector, is the generator of translation in time of the relativistic wavefunction. However, the spacelike operator $Q_{s}=s^{a} \mathcal{K}_{a}$, where $s^{a}$ is spacelike and is orthogonal to $t^{a}$, is the translation generator in space. The symmetries generated by these generators correspond to conservation laws, in which the energy and momentum conservation laws are unified into one, which is the conservation of scalar-mass energy generated by the timelike operator. For the spacelike operator, the theory suggests that there is also another conservation law that pertains to the vector-mass energy, and its physical implications are discussed in Section 7.

The above postulates form the basis of our new RQM, which is developed here for a massive and spin-zero elementary particle uniquely described by the scalar wavefunction $\phi$ for which we now obtain its wave equation.

\section{Generalized Klein-Gordon Equation and Its Solutions}

The eigenvalue equations (see Equation (11)), in general, satisfy the following secondorder Klein-Gordon (KG) eigenvalue equation

$$
\mathcal{K}_{a} \mathcal{K}^{a} \phi(\tau, \xi)=\left(m^{2}-|\boldsymbol{k}|^{2}\right) \phi(\tau, \boldsymbol{\xi})=M^{2} \phi(\tau, \xi)
$$

where $\phi=\langle\tau, \xi \mid \phi\rangle$ and $M^{2}$, whose value ranges from $-\infty$ to $\infty$, is the eigenvalue of the operator $\mathcal{K}_{a} \mathcal{K}^{a}$. The general solutions to Equation (14) for a particular $M^{2}$ is given as

$$
\phi(\tau, \boldsymbol{\xi})=A e^{-i K_{a} \xi^{a}}+B e^{-i \bar{K}_{a} \xi^{a}}=\left[A e^{-i E(\tau-\beta \cdot \xi)}+B e^{i E(\tau-\beta \cdot \mathcal{\xi})}\right] e^{i V \cdot(\mathcal{\xi}-\beta \tau)},
$$

where $K=P+V$ for the positive energy solution, and $\bar{K}=-P+V$ for the negative energy solution, which corresponds to $m \rightarrow-m$. The coefficients $A$ and $B$ are constants of integration. Note that $M^{2}$ is not the particle's mass despite its place in the KG equation. The main reason is that the particle's mass must be in a superposition of different vector-mass $k$, which does not play any role in the motion of the particle because of its spacelike origin.

It must be noted that Equation (14) is not a dynamical equation as the other wave equations; in fact, it is the extended form of the Helmholtz equation with the Minkowski metric, where the eigenvalue $M^{2}$ cannot be replaced by an operator without introducing new structures to the particle or spacetime, such as internal symmetries or extra dimensions, respectively. Therefore, accounting for spins and different generations of particles is still required, which is beyond the scope of this paper, as the main focus herein is to develop an alternative theory of RQM and present its physical implications. In the following, we discuss the relation between the completeness of Hilbert space and the causal structure of this new RQM in detail.

\section{Completeness of Hilbert Space and Causality}

After defining the basic postulates of the new RQM and presenting the resulting generalized Klein-Gordon eigenvalue equation, the completness of Hilbert space and causality must be considered. This requires the generalization of the concept of the inner product of the new state vectors in Hilbert space, which is defined as

$$
\left\langle\tau^{\prime}, \boldsymbol{\xi}^{\prime} \mid \tau, \boldsymbol{\xi}\right\rangle=\delta\left(\tau^{\prime}-\tau\right) \delta^{3}\left(\boldsymbol{\xi}^{\prime}-\boldsymbol{\xi}\right)
$$

and

$$
\left\langle m^{\prime}, \boldsymbol{k}^{\prime} \mid m, \boldsymbol{k}\right\rangle=\delta\left(m^{\prime}-m\right) \delta^{3}\left(\boldsymbol{k}^{\prime}-\boldsymbol{k}\right) .
$$

It must be noted again that a spacetime state $|\tau, \xi\rangle$ is completely defined throughout spacetime, which describes a particle that exists exactly at the point $\xi$ in space and $\tau$ in time of a local inertial observer. For such state, an observer would see the particle suddenly 
come in and out of existence, with undefined mass, energy, and momentum. Conversely, an inertial quantum state $|m, \boldsymbol{k} ; \boldsymbol{\beta}\rangle$ represents a particle of well-defined scalar-mass, vectormass, and velocity, but it does not have a well-defined spacetime position.

Using Equation (13), we write the projection of a inertial state onto a spacetime state as

$$
\langle\tau, \boldsymbol{\xi} \mid m, \boldsymbol{k} ; \boldsymbol{\beta}\rangle=\frac{e^{-i E(\tau-\beta \cdot \xi)} e^{i V \cdot(\mathcal{\zeta}-\beta \tau)}}{(2 \pi)^{2}}=\frac{e^{-i \tau(E+\beta \cdot V)} e^{i \xi \cdot(V+\beta E)}}{(2 \pi)^{2}},
$$

which is a state of definite $K$ in flat spacetime, with $\beta=P / E$ and $\tau=\xi^{0}$. Since the identity operator $\mathcal{I}$ in the spacetime basis is given by

$$
\mathcal{I}=\int \mathrm{d}^{4} \xi|\tau, \xi\rangle\langle\tau, \xi|
$$

we use Equation (18) to find

$$
\begin{array}{r}
\left\langle m^{\prime}, \boldsymbol{k}^{\prime} ; \boldsymbol{\beta}|\mathcal{I}| m, \boldsymbol{k} ; \boldsymbol{\beta}\right\rangle=\int \frac{\mathrm{d}^{4} \xi}{(2 \pi)^{4}} e^{i \tau(\Delta E+\boldsymbol{\beta} \cdot \Delta \boldsymbol{V})} e^{-i \boldsymbol{\xi} \cdot(\Delta \boldsymbol{V}+\boldsymbol{\beta} \Delta E)}= \\
\delta(\Delta E+\boldsymbol{\beta} \cdot \Delta \boldsymbol{V}) \delta^{3}(\Delta \boldsymbol{V}+\boldsymbol{\beta} \Delta E),
\end{array}
$$

where $\Delta K=K^{\prime}-K$. Note that for all allowed values of $|\boldsymbol{\beta}|<1$, Equation (20) is equivalent to Equation (17), and that the identity operator $\mathcal{I}$ is complete and the boost operator $\mathcal{U}_{\beta}$ (see Equation (10)) is unitary. Equivalently, the identity operator can be expressed in the inertial basis as

$$
\mathcal{I}=\int \mathrm{d} m \mathrm{~d}^{3} \boldsymbol{k}|m, \boldsymbol{k}\rangle\langle m, \boldsymbol{k}|,
$$

which is to show that the inertial states are also complete.

Next, we introduce two new operators, $\mathcal{T}_{\beta}$ and $\mathcal{S}$, whose definitions are motivated by their application in the next section for the construction of the path integral formulation of the new RQM. The operators are defined as

$$
\mathcal{T}_{\boldsymbol{\beta}}(m)=\int \mathrm{d}^{3} \boldsymbol{k}|m, \boldsymbol{k} ; \boldsymbol{\beta}\rangle\left\langle m, \boldsymbol{k} ; \boldsymbol{\beta}\left|=\int \frac{\mathrm{d}^{3} \boldsymbol{V}}{\gamma}\right| m, \boldsymbol{k} ; \boldsymbol{\beta}\right\rangle\langle m, \boldsymbol{k} ; \boldsymbol{\beta}|,
$$

and

$$
\mathcal{S}(\tau)=\int \mathrm{d}^{3} \xi|\tau, \xi\rangle\langle\tau, \xi|
$$

To understand the properties of these operators, we investigate the matrix elements in their respective dual basis. First, we study the properties of $\mathcal{T}_{\beta}$ with its matrix element given by

$$
\begin{array}{r}
\left\langle\tau^{\prime}, \boldsymbol{\xi}^{\prime}\left|\mathcal{T}_{\beta}\right| \tau, \boldsymbol{\xi}\right\rangle=\frac{e^{-i E(\Delta \tau-\beta \cdot \Delta \xi)}}{2 \pi \gamma} \int \frac{\mathrm{d}^{3} \boldsymbol{V}}{(2 \pi)^{3}} e^{i V \cdot(\Delta \tilde{\zeta}-\beta \Delta \tau)}= \\
\frac{e^{-i E(\Delta \tau-\beta \cdot \Delta \xi)}}{2 \pi \gamma} \delta^{3}(\Delta \xi-\beta \Delta \tau) .
\end{array}
$$

Note that we did not integrate over the scalar-mass $m$. This operator is defined for a particle of definite scalar-mass. The immediate interpretation of this result is that this is exactly the causal path of the particle traveling between the two spacetime states, where $\Delta \xi=\xi^{\prime}-\xi$. For a particle of definite mass $m$, the result shows that the particle must be stationary in space when its velocity $\beta=0$, while the complex phase evolves over proper time of the particle, i.e.,

$$
\left\langle\tau^{\prime}, \boldsymbol{\xi}^{\prime}\left|\mathcal{T}_{\beta=0}\right| \tau, \xi\right\rangle=\frac{e^{-i m \Delta \tau}}{2 \pi} \delta^{3}(\Delta \xi)
$$


Alternatively, when $\beta \neq 0$, the result (24) is nonzero if, and only if, the states are causally separated, i.e., the path traced out by the particle must stay within the light cone. The complex phase difference between the final and initial states is given by the complex exponential prefactor. Remarkably, this is simply the quantum path of a free point particle and the matrix element is just the phase evolution along the path of propagation. This operator is the propagation operator for a particle of mass $m$ moving from $|\tau, \xi\rangle$ to $\left|\tau^{\prime}, \boldsymbol{\zeta}^{\prime}\right\rangle$; however, it is different from the time evolution operator in QM because the time dependence is already contained in the spacetime states $|\tau, \xi\rangle$, while the operator $\mathcal{T}_{\beta}$ simply connects the two spacetime states by imparting the particle with a particular mass $m$ moving at the velocity $\beta$.

In fact, the particle itself can be associated with the operator $\mathcal{T}_{\beta}$. However, as we recall the general solution to the KG eigenvalue equation in Equation (15), we see that it is a linear combination of both positive and negative energy terms; therefore, we must also form a linear combination of the operator $\mathcal{T}_{\beta}$ with its negative energy counter part, namely $m \rightarrow-m$, to describe a particle of mass $m>0$.

Let us refer to the linear combination

$$
\mathcal{P}_{\beta}(m)=a_{\beta}^{+} \mathcal{T}_{\beta}(m)+b_{\beta}^{-} \mathcal{T}_{\beta}(-m),
$$

as the 'particle operator' that creates a particle or destroys an antiparticle of mass $m$, and its corresponding mass conjugation

$$
\mathcal{P}_{\boldsymbol{\beta}}^{\star}(m)=a_{\boldsymbol{\beta}}^{-} \mathcal{T}_{\boldsymbol{\beta}}(-m)+b_{\boldsymbol{\beta}}^{+} \mathcal{T}_{\boldsymbol{\beta}}(m),
$$

as the 'antiparticle operator' that creates an antiparticle or destroys a particle of mass $m$.

Here, we have promoted the numerical coefficients to creation and annihilation operators that act on Fock space of occupancy number basis, $|n\rangle$, and defined the mass conjugation of the operator $\mathcal{T}_{\beta}$ as

$$
\mathcal{T}_{\beta}^{\star}(m)=\mathcal{T}_{\beta}(-m),
$$

and

$$
\left(a_{\beta}^{+}\right)^{\star}=a_{\beta}^{-} .
$$

By promoting the coefficients to operators, the spacetime states must also be promoted to the spacetime Fock's states $|\tau, \xi ; n\rangle=|\tau, \xi\rangle \otimes|n\rangle$, such that $|\tau, \xi ; 0\rangle$ represents the vacuum state and $|\tau, \xi\rangle$ represents single particle state. Thus, we have

$$
\left\langle\tau^{\prime}, \xi^{\prime}\left|\mathcal{P}_{\beta}\right| \tau, \xi ; 0\right\rangle=\left\langle\tau^{\prime}, \xi^{\prime}\left|\mathcal{T}_{\beta}\right| \tau, \xi\right\rangle
$$

Finally, we have the matrix element of the operator $\mathcal{S}$ expressed as follows

$$
\left\langle m^{\prime}, \boldsymbol{k}^{\prime} ; \boldsymbol{\beta}|\mathcal{S}| m, \boldsymbol{k} ; \boldsymbol{\beta}\right\rangle=\frac{e^{-i \tau(\Delta E+\boldsymbol{\beta} \cdot \Delta \boldsymbol{V})}}{2 \pi} \delta^{3}(\Delta \boldsymbol{V}+\boldsymbol{\beta} \Delta E),
$$

which is the spatial inner product of two inertial states at time $\tau$. The $\mathcal{S}$ operator specifies a local inertial frame, in which the inner product can be calculated, or the spatial projection of one particle state onto another. As shown by Equation (31), the projection is given for two states of different four-mass vectors with the same velocity. This may be interpreted as the transition amplitude of a particle changing its mass as it propagates, or as a way to project states of the same masses but different velocities. In the second case, the result would be the transition amplitude of the particle changing its velocity. In either case, the particle is undergoing an interaction, where the former could describe the particle decay process and the latter for a particle experiencing a force. However, in this paper we consider only free particles. 
In the next section, the pair $\mathcal{S}$ and $\mathcal{T}_{\beta}$ is used to recover the relativistic classical Lagrangian of a free particle by following the Feynman path integral formulation of QM. Our goal is to obtain a consistent relativistic path integral and, ultimately, confirm the validity of our reformulated theory of RQM.

\section{Path Integral Formulation}

We now present the path integral formulation of the developed RQM by deriving the transition amplitude for a particle with a definite scalar-mass $m$. The integration is performed over all possible $|\boldsymbol{\beta}|<1$, or equivalently, $|\gamma \boldsymbol{\beta}|<\infty$, since a quantum particle can take any path and the velocity integration is bounded because the particle is massive. Then, using the operator $\mathcal{T}_{\beta}$ from previous section (see Equation (22)), the general transition operator $\mathcal{T}$ is given by

$$
\mathcal{T}(m)=\int_{|\gamma \boldsymbol{\beta}|<\infty} \frac{\mathrm{d}^{3}(\gamma \boldsymbol{\beta})}{\gamma} \mathcal{T}_{\boldsymbol{\beta}}(m)=\int_{|\boldsymbol{\beta}|<1} \mathrm{~d}^{3} \boldsymbol{\beta} \gamma^{2} \mathcal{T}_{\boldsymbol{\beta}}(m) .
$$

The presence of the factor $\gamma$ is to keep the integration Lorentz-invariant. Using the particle operator to create a particle of mass $m$ out of the vacuum as shown in Equation (30), we integrate Equation (24) to obtain the relativistic quantum transition amplitude

$$
\begin{array}{r}
\left\langle\tau^{\prime}, \boldsymbol{\zeta}^{\prime}|\mathcal{T}| \tau, \boldsymbol{\zeta}\right\rangle=\int_{|\beta|<1} \mathrm{~d}^{3} \beta \frac{e^{-i E(\Delta \tau-\beta \cdot \Delta \xi)}}{2 \pi \gamma^{-1}} \delta^{3}(\Delta \xi-\beta \Delta \tau)= \\
\frac{e^{-i m \Delta \bar{\tau}}}{2 \pi|\Delta \bar{\tau}|} \Theta\left(1-\left|\frac{\Delta \xi}{\Delta \tau}\right|^{2}\right),
\end{array}
$$

where

$$
\Delta \bar{\tau}=\Delta \tau \sqrt{1-\left|\frac{\Delta \xi}{\Delta \tau}\right|^{2}}
$$

and the function $\Theta(x)$ is the Heaviside step function, whose presence is necessary to prevent the particle from propagating faster than the speed of light.

The Feynman path integral formalism describes the procedure of calculating the propagator matrix element $G\left(\xi_{F} ; \xi_{I}\right)$, also known as Green's function, of a free particle moving from $\xi$ to $\xi^{\prime}$. This is achieved by integrating the Feynman propagation phase factor over all possible timelike paths that the particle could take. To obtain this operator, firstly we subdivide spacetime phase space into $N$ slices of spacelike hypersurfaces $\Sigma_{n}$ and the corresponding dual surfaces $\Omega_{n}$, where $\xi_{n} \in \Sigma_{n}$ and $k_{n} \in \Omega_{n}$. Each surface $\Sigma_{n}$ is spatial surface that represents a time slice in spacetime where the particle could be found at time $\tau_{n}$. The integration over this surface is represented by the operator $\mathcal{S}_{\tau_{n}}$.

Then, we connect the different hypersurfaces using the summation over all world lines that the particle could take. This is achieved by including the transition operators $\mathcal{T}_{n}$ of a fixed scalar-mass in between $\mathcal{S}_{\tau_{n}}$. Lastly, we take the continuum limit as $N \rightarrow \infty$ and $\Delta \tau_{n} \rightarrow 0$. This construction gives us the following the time-ordered products

$$
\begin{array}{r}
G_{N}\left(\xi_{F}, \xi_{I}\right)=\left\langle\xi_{F}\left|\mathcal{U}_{N}\right| \xi_{I}\right\rangle\left\langle\xi_{N}\left|\mathcal{T}_{N} C_{N-1} \mathcal{S}_{\tau_{N-1}} \mathcal{T}_{N-1} \cdots C_{n} \mathcal{S}_{\tau_{n}} \mathcal{T}_{n} \cdots C_{1} \mathcal{S}_{\tau_{1}} \mathcal{T}_{1}\right| \xi_{0}\right\rangle \\
=\left[\prod_{n=1}^{N-1} \int_{\Sigma_{n}} \mathrm{~d}^{3} \xi_{n} C_{n}\right]\left[\prod_{j=1}^{N}\left\langle\xi_{j}\left|\mathcal{T}_{j}\right| \xi_{j-1}\right\rangle\right],
\end{array}
$$

where $\tau_{n}>\tau_{n-1}$ and $C_{n}$ is some normalization constant to be determined. The matrix elements of $\mathcal{T}_{j}$ are given by Equation (33). Thus, we have

$$
\left\langle\xi_{j}\left|\mathcal{T}_{j}\right| \xi_{j-1}\right\rangle=\frac{e^{-i m \Delta \bar{\tau}_{j}}}{2 \pi \Delta \bar{\tau}_{j}} \Theta\left(1-\left|\frac{\Delta \xi_{j}}{\Delta \tau_{j}}\right|^{2}\right)
$$


which leads to

$$
G_{N}\left(\xi_{F}, \xi_{I}\right)=\left[\prod_{n=1}^{N-1} \int \mathrm{d}^{3} \xi_{n} C_{n}\right] \prod_{j=1}^{N}\left(\frac{e^{-i m \Delta \bar{\tau}_{j}}}{2 \pi \Delta \bar{\tau}_{j}}\right)
$$

where $\Delta \xi_{j}=\xi_{j}-\xi_{j-1}$. We have omitted the Heaviside functions for legibility and

$$
\Delta \bar{\tau}_{j}=\Delta \tau_{j} \sqrt{1-\left|\frac{\Delta \xi_{j}}{\Delta \tau_{j}}\right|^{2}}
$$

With the endpoints fixed and the velocity bounded by the speed of light, we take the limit $N \rightarrow \infty$ and obtain

$$
G\left(\xi_{F}, \xi_{I}\right)=\int \mathrm{D} \xi(\bar{\tau}) C e^{i S}
$$

where the integral is appropriately normalized by $C$. Then, the path-integral measure in the continuum limit is

$$
\mathrm{D} \xi(\bar{\tau})=\lim _{N \rightarrow \infty} \prod_{n=1}^{N-1} \mathrm{~d}^{3} \xi_{n}
$$

The above results demonstrate that the classical action of a relativistic free particle is correctly recovered from the path-integral formulation, with the action given by

$$
S=-m \int_{\bar{\tau}_{I}}^{\bar{\tau}_{F}} \mathrm{~d} \bar{\tau}
$$

where $\bar{\tau}$ is the proper time of a relativistic particle. We may finally define the evolution operator $\mathcal{U}$ that acts on states in spacetime basis as

$$
\mathcal{U}(\tau)=\lim _{N \rightarrow \infty} \mathcal{T}_{N} \prod_{n=1}^{N-1} C_{n} \mathcal{S}_{\tau_{n}} \mathcal{T}_{n}
$$

Now, developing an analytical method to evaluate the relativistic path integral given in Equation (39) is out the scope of this paper. Nonetheless, for a free particle this problem is equivalent to showing that it reduces to the result (see Equation (33)) for the operator $\mathcal{T}$.

\section{Physical Implications}

The main differences between the standard RQM and the reformulated RQM presented in this paper are summarized in Table 1. Let us now briefly comment on the concepts given in Table 1. As a consequence of symmetry between the timelike and spacelike intervals, the four momentum is no longer confined to the light cone, and that the theory requires both the scalar-mass and vector-mass; although only scalar-mass is observable, the four-mass vector labels the mass state of the particle. The (linear) velocity is a parameter of the Lorentz boost. The reformulation makes an explicit distinction between the vector-mass and the velocity vector. The quantum nature of the particle is encoded in the superposition of different vector-masses and velocities. The proper time and distance are defined relative to an inertial observer and the reformulation redefines the Heisenberg uncertainty principles with the new conjugate variable pairs. Furthermore, the generalized KG equation obtained in this paper is not a wave equation but an eigenvalue equation. Unlike the square of the scalar-mass in the conventional theory being a constant, $M^{2}$ is an eigenvalue. On the one hand, the conservation of timelike component incorporates both the conservation of momentum and energy; on the other hand, the conservation of spacelike component could be related to the conservation of quantum information and responsible for the nonlocal quantum effects. Most importantly, the reformulated theory reproduces the well-known relativistic Lagrangian of a classical particle for the path integral formulation. 
Table 1. Differences between the standard and new RQM.

\begin{tabular}{lll}
\hline Concepts & Standard RQM & New RQM \\
\hline Four momentum & Timelike & Timelike and spacelike \\
Invariant mass & Scalar & Scalar and vector \\
Particle's velocity & Implicit & Explicit \\
Conjugate variables & Energy-time & Scalar-mass-proper time \\
Conjugate variables & Momentum-position & Vector-mass-proper distance \\
Conserved quantities & Timelike components & Timelike and spacelike components \\
Path integral & All trajectories & Trajectories inside the light cone \\
\hline
\end{tabular}

The reformulated RQM presented in this paper takes into account the spacelike eigenvalues of the linear momentum, in addition to its timelike eigenvalues. The timelike and spacelike eigenvalues are associated with the scalar-mass $m$ and vector-mass $k$, respectively, with the latter being a new physical concept. The theory demands that $m$ and vector-mass $k$ are independent observable quantities. However, in practice only $m$ can be observed since all observers and their experiments are confined to their local light cone. On the other hand, $k$ does not describe the particle's causal motion to an inertial observer, therefore, it cannot be measured experimentally because of its spacelike nature outside of the light cone. Despite this spacelike nature of $k$, the developed theory is not a hidden-variable theory and it preserves causality.

Moreover, the presence of vector-mass does not affect particle's probability amplitude because $k$ is integrated out of the complex phase when the operator $\mathcal{T}_{\beta}$ is projected onto the spacetime states. The result is that the complex phase that involves $k$ becomes a delta function representing the localization of the particle in spacetime. The remaining complex phase contains the scalar-mass and the proper time, which becomes the probability amplitude of the particle for a particular path that it takes as it is shown by the path integral formulation (see Section 6). Other important physical implications of the presence of the vector-mass in the theory include nonlocality, a novel picture of vacuum, and some modifications of the uncertainty principle, and they are now discussed.

There is a large body of literature on the subject of nonlocality and imcompleteness in QM and the subsequent developments triggered by the original Einstein, Podolsky and Rosen paper [36], and Bell's work [37-39]. Bell showed that hidden-variable theories must be nonlocal, in order to be in agreement with the empirically verified predictions of $\mathrm{QM}$, and that nonlocality of QM cannot be attributed to incompletness [40]. The work on nonlocality in QM initiated formulations of different nonlocal theories of QFT. In the earlier work summarized in [41], the nonlocal theories were constructed by either introducing a new universal length that allows for a slight violation of locality on small distances, or defining a constant that characterizes the domain of nonlocal interactions. In more recent work, nonlocal QFT for scalar fields were constructed [42,43].

In the RQM theory presented in this paper, the scalar-mass is responsible for the time evolution of the wavefunction, similar to standard RQM; however, the vector-mass affects only the spatial distribution of the wavefunction, which makes our theory a fully symmetric theory and, thus, different than those local and nonlocal theories previously constructed [41-43]. We identify the spacelike nature of the wavefunction spatial distribution with nonlocality in our theory and suggest that due to the presence of vector-mass, the particle may potentially interact nonlocally with another particle by overlapping the spatial distributions of their wavefunction. Since our theory considers only one free, massive, and spin-zero elementary particle, such nonlocally interacting particles are out of the scope of this paper.

In this reformulated RQM, the scalar-mass is responsible for the the particle's worldline being within the light cone. Moreover, a particle of definite mass and position in space must be in a superposition of different vector-masses, which means that $k$ does not play any role in the motion of the particle. As a result, $M^{2}$ given by Equation (4) is not the 
mass of the particle and the theory allows only for particles with positive $m^{2}$ term, which prevents tachyons and tachyonic fields [31,32] from being defined in this theory.

The vector-mass, as the spacelike component responsible for the nonlocal behavior of quantum particles, is the true conjugate variable of position in $\mathbb{R}^{3}$ space, thus, the canonical commutation relations given by Equation (5) can be used to write the uncertainty principle in the following form

$$
\prod_{i=1}^{3} \Delta \xi^{i} \cdot \Delta k^{i} \geq 1
$$

There is also uncertainty of the scalar-mass due to the presence of $k$ and this uncertainty is inversely proportional to particle's lifetime, then the resulting uncertainty relation becomes

$$
\Delta \tau \Delta m \geq 1,
$$

and it describes virtual particles that are present in this reformulated RQM; note that according to our theory only particles with infinite lifetime are stable and observable. The presence of virtual particles in the theory leads to a novel picture of vacuum that is now briefly described.

From the point of view of RQM and QFT, vacuum is defined as the state of lowest energy filled with virtual particles spontaneously coming out of the void and disappearing in it (e.g., [44,45]), with the notion that known elementary particles may form pairs with their antiparticles and become virtual on the time and energy scales corresponding to these particles (e.g., [46-48]). The theory presented in this paper and its uncertainty relation given by Equation (44) shows that masses of virtual particles do not have to be limited by experimentally established masses of known elementary particles, as virtual particles may have any mass, including masses of all known particles, which are required by this theory to be stable and detectable if, and only if, their lifetimes are infinititely long.

\section{Conclusions}

Standard RQM that is based on the timelike intervals is reformulated by taking into account both the timelike and spacelike intervals. The presence of the spacelike intervals in the reformulated RQM modifies its linear momentum by including its spacelike eigenvalues, in addition to timelike eigenvalues. The theory is developed for a free, massive, and spin-zero elementary particle in the Minkowski spacetime, where the particle is called elementary if its wavefunction transforms as one of the irreps of the Poincaré group. The STR energy-momentum relationship of standard RQM with its scalar-mass is modified by an additional term with its vector-mass, which only affects the spatial distribution of particle's wavefunction. The physical consequences of the presence of the vector-mass in the energy-momentum relationship are far reaching, starting with the modification fo the mass term in the Klein-Gordon equation, and then leading to nonlocality, some modifications of the uncertainty principle, and a novel picture of vacuum. Moreover, the developed RQM theory preserves causality, is self-consistent, and is formulated using the path integral formalism, which correctly reproduces the classical action of a relativistic particle; however, the theory does not allow for tachyons and tachyonic fields. The theory is not a hidden-variable theory; its physical interpretation is consistent with the Copenhagen interpretation of $\mathrm{QM}$, and preserves causality.

Author Contributions: Conceptualization, B.D.T. and Z.E.M.; methodology, B.D.T.; validation, B.D.T. and Z.E.M.; formal analysis, B.D.T.; investigation, B.D.T. and Z.E.M.; writing—original draft preparation, B.D.T. and Z.E.M.; writing-review and editing, B.D.T. and Z.E.M. All authors have read and agreed to the published version of the manuscript.

Funding: This research received no external funding.

Institutional Review Board Statement: Not applicable.

Informed Consent Statement: Not applicable. 


\section{Data Availability Statement: Not applicable.}

Acknowledgments: We are grateful to the two anonymous referees for their comments and suggestions that allowed us to improved the original version of this paper. We also thank Lesley Vestal for reading our manuscript and commenting on it.

Conflicts of Interest: The authors declare no conflict of interest.

\section{References}

1. Greiner, W. Relativistic Quantum Mechanics; Springer: Berlin/Heidelberg, Germany, 1990.

2. Gross, F. Relativistic Quantum Mechanics and Field Theory; John Wiley \& Sons, Inc.: New York, NY, USA, 1993.

3. Klein, O. Quantentheorie und fünfdimensionale Relativitätstheorie. Z. Phys. 1926, 37, 895. [CrossRef]

4. Gordon, W. Der Comptoneffekt nach der Schrödingerschen Theorie. Z. Phys. 1926, 40, 117. [CrossRef]

5. Dirac, P.A.M. The quantum theory of the electron. Proc. R. Soc. Lond. 1928, 117, 610.

6. Proca, A. Sur la théorie ondulatoire des électrons positifs et négatifs. J. Phys. Radiium 1936, 7, 347. [CrossRef]

7. Rarita, W.; Schwinger, J. On a Theory of Particles with Half-Integral Spin. Phys. Rev. 1941, 60, 61. [CrossRef]

8. Ryder, L.W. Quantum Field Theory; Cambridge University Press: Cambridge, UK, 1985.

9. Frampton, P.H. Gauge Field Theories; John Wiley \& Sons, Inc.: New York, NY, USA, 2000.

10. Kim, Y.S.; Noz, M.E. Theory and Applications of the Poincaré Group; Reidel: Dordrecht, The Netherlands, 1986.

11. Bergmann, V.; Wigner, E. Group theoretical adiscussion of relativistic wave equations. Proc. Natl. Acad. Sci. USA 1948, 34, 211. [CrossRef]

12. Wigner, E.P. On unitary representations of the inhomogeneous Lorentz group. Ann. Math. 1939, 40, 149. [CrossRef]

13. Fushchich, W.I.; Nikitin, A.G. Symmetries of Equations of Quantum Mechanics; Allerton Press: Allerton, IL, USA, 1994.

14. Fry, J.L.; Musielak, Z.E. Fundamental theories of waves and particles formulated without classical mass. Ann. Phys. 2010, 325, 2668. [CrossRef]

15. Fry, J.L.; Musielak, Z.E.; Chang, T. Relativistic wave and particle mechanics formulated without classical mass. Ann. Phys. 2011, 326, 1972. [CrossRef]

16. Flato, M.; Sternheimer, D.; Sternheimer, J.; Vigier, J.P.; Wataghin, G. On the masses of nonstrange pseudoscalar mesons and the generalized Klein-Gordon equation. Nuovo Cim. 1966, 42, 431-434. [CrossRef]

17. Bollini, C.G.; Giambiagi, J.J. Generalized Klein-Gordon equation in d dimensions in supersymmetry. Phys. Rev. D 1985, 32, 3316. [CrossRef]

18. Nieto, J.L.; Lu, J.A.; Vergara, J.D. A generalized Klein-Gordon equation form a reparametrized Lagrangian. Phys. Let. A 1996, $219,150$.

19. De Haas, E.P.J. A Generalized Klein-Gordon Equation with a Closed System Condition for the Dirac-Currant Probability/Field Tensor. Available online: https://vixra.org/abs/1810.0349 (accessed on 2 May 2021).

20. Leiter, D.; Szamosi, G. Pseudoscalar mass and its relationship to conventional scalar mass in relativistic Dirac theory of the electron. Lett. Nuovo Cim. 1972, 5, 814. [CrossRef]

21. Barut, A.O. The mass of muon. Phys. Lett. 1978, 73B, 310. [CrossRef]

22. Pfister, W. Mixed-symmetry solutions of generalized three-particle Bargmann-Wigner equations in the strong-coupling limit. Nuovo Cim. A 1995, 108, 1427. [CrossRef]

23. Kruglov, S.I. On the Hamiltonian form of generalized Dirac equation for fermions with two mass states. Elect. J. Theor. Phys. 2006, 3, 11 .

24. Kruglov, S.I. Modified Dirac equation with Lorentz invariance violoation and its solutions for particles in an external magnetic field. Phys. Let. B 2012, 718, 228. [CrossRef]

25. Nozari, K. Generalized Dirac equation and its symmetries. Chaos Solitons Fractals 2007, 32, 302. [CrossRef]

26. Dvoeglazov, V.V. The Bargmann-Wigner formalism for higher spins (up to 2). Int. J. Mod. Phys. 2011, $2,121$.

27. Huegele, R.; Musielak, Z.E.; Fry, J.L. Generalized Dirac and Klein-Gordon equations for spinor wavefunctions. Adv. Stud. Theor. Phys. 2013, 7, 825. [CrossRef]

28. El-Nabulsi, R.A. Generalized Klein-Gordon and Dirac equations from nonlocal kinematic approach. Z. Naturforsch. 2016, 71, 817. [CrossRef]

29. Nierdele, J.; Nikitin, A.G. Relativistic wave equations for interacting, massive particles with arbitrary hal-integer spins. Phys. Rev. D 2001, 64, 125013 .

30. Watson, T.B.; Musielak, Z.E. Chiral symmetry in Dirac equation and its effecst on neutrino masses and dark matter. Int. J. Mod. Phys. A 2020, 35, 2050189. [CrossRef]

31. Recami, E. Classical tachyons and possible applications. Riv. Nuovo Cim. 1986, 9, 1. [CrossRef]

32. Schwartz, C. Toward a quantum theory of tachyon fields. Int. J. Mod. Phys. A 2016, 31, 1650041. [CrossRef]

33. Bohm, D. Quantum Theory; Prentice-Hall, Inc.: New York, NY, USA, 1951.

34. Bohr, N. Can quantum-mechanical description of physical reality be considered complete? Phys. Rev. 1935, 48, 696. [CrossRef]

35. Faye, J. Copenhagen Interpretation of Quantum Mechanics, in Stanford Encyclopedia of Philosophy; Zalta, E., Ed.; Methaphysics Research Laboratory; Stanford University: Stanford, CA, USA, 2019. 
36. Einstein, A.; Podolsky, B.; Rosen, N. Can quantum-mechanical description of physical reality be considered complete? Phys. Rev. 1935, 47, 777. [CrossRef]

37. Bell, J. On the Einstein Podolsky Rosen paradox. Physics 1964, 1, 195. [CrossRef]

38. Hughes, R.I.G. The Structure and Interpretation of Quantum Mechanics; Harvard University Press: Cambridge, MA, USA, 1992.

39. Maudlin, T. Quantum Non-Locality and Relativity; Blackwell Publishers Ltd.: Oxford, UK, 2002.

40. Norsen, T. EPR and Bell locality. arXiv 2005, arXiv:quant-ph/0408105v3.

41. Namsrai, K. Foundation of the Nonlocal Model of Quantized Fields, in Nonlocal Nonlocal Quantum Field Theory and Stochastic Quantum Mechanics; Reidel Publishing Company: Dordrecht, The Netherlands, 1986; pp. 3-45.

42. Morgan, P. A classical perspective on nonlocality in quantum field theory. arXiv 2001, arXiv:quant-ph/0106141v4.

43. Bernard, M.; Guskov, V.A.; Ivanov, M.G.; Kalugin, A.E.; Ogarkov, S.L. Nonlocal scalar quantum field theory-Functional integration, basis functions representation and strong coupling expansion. Particles 2019, 2, 385. [CrossRef]

44. Wilczek, F. Quantum field theory. Rev. Mod. Phys. 1999, 71, 83. [CrossRef]

45. Boi, L. The Quantum Vacuum; The Johns Hopkins University Press: Baltimore, MD, USA, 2011.

46. Leinaas, J.M.; Olaussen, K. Vacuum structure and the fermion-boson transitions. Phys. Rev. D 1986, 34, 2483. [CrossRef]

47. Haisch, B.; Rudea, A.; Dobyns, Y. Inertial mass and the quantum vacuum fields. Ann. Phys. 2001, 10, 393. [CrossRef]

48. Bogolubov, N.N., Jr.; Prykarpatsky, A.; Taneri, U. The vacuum structure, special relativity theory and quantum mechanics revisited: A field theory-no-geometry approach. Theor. Math. Phys. 2009, 160, 1079. [CrossRef] 